\title{
La estructura retórica de consentimientos informados usados en tesis y trabajos de grado de odontología en español
}

\author{
Jesús Arias
}

Universidad de Los Andes

\section{André Caldera}

Universidad de Los Andes

Oscar Alberto Morales ${ }^{1}$

Universidad de Los Andes y Universidad de los Hemisferios

Facultad de Odontología, Departamento de Investigación. Mérida, Estado Mérida (Venezuela)

Bexi Perdomo

Universidad de Los Andes

Daniel Cassany

Universitat Pompeu Fabra

Elix Izarra

Universidad de Los Andes

\section{RESUMEN}

Introducción: El consentimiento informado (CI) consiste en certificar que el paciente ha recibido información sobre su condición, las alternativas de tratamientos, sus efectos y consecuencias derivadas del procedimiento clínico. Su uso en la clínica odontológica ha sido ampliamente investigado, pero no en investigación. Objetivo: describir la estructura retórica del CI usado en tesis/trabajos de grado presentados en español en Latinoamérica y España. Metodología: Se analizaron la estructura retórica, secciones y movimientos y la organización de 60 documentos de CI, mediante el análisis de género y entrevistas a informantes expertos. Resultados: Se encontraron cuatros secciones sucesivas: (a) información institucional; (b) el título consentimiento informado; (c) cuerpo del documento: (1) identificación de personas involucradas, (2) descripción de la investigación, (3) afirmación de haber recibido información, (4) de haberla comprendido, (5) ratificación del consentimiento; y (d) cierre. Conclusiones: se sugiere incluir en el CI los elementos y secciones encontradas en este estudio.

Palabras clave: consentimiento informado, estructura retórica, tesis/trabajo de grado, análisis de género, Odontología.

\section{ABSTRACT}

Introduction: Informed consent (IC) consists in certifying that the patient has received information about their condition, treatment alternatives, the effects and consequences derived from the clinical procedure. Its use in the dental clinic practice has been widely investigated, but not in research. Objective: to describe the rhetorical structure of IC used in thesis and dissertations in Spanish in Latin America and Spain. Methods: The rhetorical structure, sections and movements and the organization of $60 \mathrm{CI}$ documents were analyzed, through genre analysis and interviews with expert informants. Results: Four successive sections were found: (a) 
institutional information; (b) the title informed consent; (c) body of document, which includes: (1) identification of persons involved, (2) description of the investigation, (3) statement of having received information, (4) having understood it, (5) ratification of consent; and (d) closure. Conclusions: it is suggested to include in the IC the elements and sections found in this study.

Key words: Informed consent, rhetorical structure, thesis/dissertation, genre analysis, Dentistry.

\section{Introducción}

El modelo clásico de atención, en el que el profesional de la salud toma las decisiones y actúa según su criterio sin considerar la opinión de su paciente, ha ido cambiando progresivamente. En la actualidad, se incorpora al paciente en el proceso de decidir las acciones a seguir y se le hace corresponsable de su tratamiento (Quintero et al., 2016). Este cambio se debe, por una parte, al reconocimiento de la autonomía e integridad del paciente (Olufowote, 2008), sus expectativas y necesidades; por otra parte, a que se ha demostrado que la buena relación médico-paciente ayuda a la adhesión de los tratamientos y, por lo tanto, a su éxito(Oliva et al., 2011).

El nuevo modelo exige al profesional informar a su paciente sobre todo lo referente a su salud y al tratamiento y con ello surge la necesidad de hacer uso del consentimiento informado (en adelante, CI) (Faden y Beauchamp, 1986), un contrato, oral o escrito, que convienen las partes involucradas para iniciar un plan de tratamiento, el cual debe darse en un lenguaje adecuado para que el paciente pueda comprender los beneficios que obtendrá, las obligaciones que asume y los riesgos que corre (Abou, 2010). Se considera como un acto de expresión de dos voluntades (odontólogo y paciente), que intervienen en un procedimiento clínico de cualquier índole, ambas debidamente civilmente hábiles, competentes, autónomas, en pro de tomar las mejores decisiones sobre la base de las alternativas propuestas (Garbin et al., 2007). Roges et al. (2010) lo definen como un proceso, producto de un trabajo permanente de comunicación entre el equipo de salud y el paciente

El uso del CI ha convertido la práctica odontológica en un proceso informativo y deliberativo; por ello, el paciente tiene el derecho de consentir o rehusar propuestas de carácter preventivo, diagnóstico o terapéutico que puedan afectar la integridad físico-psíquica o social. El profesional, por su parte, debe respetar la decisión del paciente, continuar o terminar el procedimiento clínico o su participación en la investigación (Doyal y Cannell, 1995; Marchi y Sztajn, 1998; Muñoz et al., 1998; Correia y Silva, 1999; Grez, 1999; Baú, 2000; Duque, 2011).

Asimismo, en la investigación en la cual se experimenta con seres humanos su aplicación es obligatoria, para honrar los principios de autonomía, respeto e integridad de los pacientes (Olufowote, 2008). El ser humano tiene el derecho y la capacidad de elegir y actuar con autonomía y libertad, es decir, determinar su propia norma; autónomamente tiene la libertad de elegir, aplicando su propio razonamiento y una vez analizados los aspectos negativos y positivos, determinará qué conducta seguir (Garbin et al., 2007, 2009; Pascucci y Travieso, 2008).

Al analizar la ética en el ejercicio de la odontología, Pascucci y Travieso, 2008) identificaron algunos criterios que se deben cumplir para la experimentación en seres humanos, sea terapéutica (que busca aplicar correctivos, sanar, o eliminar algún daño o patología) o no (que tiene como finalidad investigar, verificar hipótesis). Sugieren que se respete el libre consentimiento y la autonomía del paciente, que se le permita actuar conforme a su voluntad, que pueda expresarse y que, si el paciente no lo puede hacer, se debe recurrir a su representante legal.

Esto es de gran importancia para todos los involucrados: para el paciente, pues mejora el resultado del tratamiento y queda más satisfecho; para los familiares, especialmente de menores y personas que no se pueden valer por sí mismos, pues permite involucrarse más en los procedimientos clínicos, y para los especialistas 
prestadores de servicios, puesto que niveles altos de involucramiento y satisfacción del paciente favorecen el éxito del procedimiento clínico (Olufowote, 2008; Oliva et al., 2011).

En el ámbito internacional, la investigación sobre el CI se ha centrado en analizar la frecuencia de uso en la práctica clínica: en Medicina, resaltan estudios en Suecia (Brehaut et al., 2015), España (Ramírez et al., 2013), Pakistán (Tahir et al., 2009), India (Gupta et al., 2014; Nouri y Rudd, 2015), Estados Unidos (Falagas et al., 2009), Canadá (Firdouse et al., 2017), Costa Rica (Rodríguez, 2012), Cuba (García et al., 2011), Chile (Oppliger y Bascuñan, 2011), México (Muñoz et al., 2008), Perú (Málaga et al., 2007; Castro et al., 2010) y Colombia (Visbal, 2009; Escobar y Novoa, 2016). También, aunque menos numerosos, se encontraron estudios en el campo de la Odontología en Nigeria (Oneyeaso y Utomi, 2007; Taiwo y Kass, 2009), Cuba (Quintana, 2009), Chile (Palomer, 2009), Brasil (Garbin et al., 2007; Maluf et al., 2007; Garbin et al., 2007, 2009), Estados Unidos (Pawla et al., 2015), Paraguay (Britez, 2001; Guirland, 2011), Perú (Alfaro y García, 2011), Venezuela(Ariasgago y Marasso, 2014; Brito y Corral, 2014; Ferro, 2014; Quintero et al. 2016) y Argentina (Miguel y Zemel, 2006).

La comprensión del CI por parte del paciente odontológico también ha sido objeto de estudio (Muñoz et al., 2008; Visbal, 2009; Guirland, 2011; Ramírez et al., 2013). En cuanto a las características del documento, solo se reportaron estudios en el área de medicina (Garbin et al., 2007; Málaga et al., 2007; Castro et al., 2010; Brehaut et al., 2015; Escobar y Novoa, 2016). En relación con el uso el ámbito de la investigación, Ariasgago y Marasso (2014) señalan que aproximadamente el 68\% de las investigaciones odontológicas venezolanas que involucran seres humanos no mencionan la correcta aplicación del CI, por lo que los aspectos bioéticos necesarios no se incluyen en las publicaciones odontológicas.

Sin embargo, hasta la fecha, el CI no ha sido estudiado desde la perspectiva discursiva (Alufowote, 2008). En la literatura revisada no hemos hallado estudios sobre el CI empleado en el ámbito de la investigación clínica odontológica hispanoamericana. Por ello, este artículo pretende describir, con fines didácticos, la estructura retórica ${ }^{2}$ del CI incluido en tesis y trabajos de grado de odontología hispanoamericana, presentados entre el 2000 y 2016, disponibles gratuitamente en los repositorios universitarios, con texto completo.

Como una primera aproximación a la estructura retórica del consentimiento informado empleado en la investigación odontológica, los resultados de esta investigación pueden contribuir a mejorar varios parámetros del género (estructura, registro, datos aportados, etc.) para que el documento sea lo suficientemente informativo, pueda comprenderse fácilmente y refleje el consenso entre las partes respetando su naturaleza jurídica contractual. Esto puede ser incluido en los programas de enseñanza de la investigación y la escritura académica en Odontología.

\section{Metodología}

2 La estructura retórica se estudia en tres niveles: movimientos retóricos, pasos y realizaciones lingüísticas. Un movimiento es cada uno de los fragmentos textuales en los que están subdivididas las secciones retóricas de un género discursivo, caracterizados por compartir un mismo contenido semántico y una misma función comunicativa. Los pasos, en cambio, son las expresiones mediante las cuales se pueden descomponer, en que se pueden realizar concretamente los movimientos para lograr sus propósitos. Por su parte, la realización lingüística es un enunciado concreto, tomado literalmente del documento, mediante el cual se pueden expresar los pasos (Skelton, 1994). 
Esta es una investigación descriptiva, pues persigue describir la estructura retórica de los CI usados en tesis y trabajos de grado de Odontología hispanoamericana.

Por otro lado, se emplea la teoría y la metodología del análisis de género discursivo. En este contexto, adoptamos un enfoque multidimensional de múltiples perspectivas, aplicado a la enseñanza del discurso académico. Recurrimos de forma complementaria al análisis textual, cualitativo y cuantitativo, y al análisis contextual mediante entrevistas a informantes expertos (Bhatia, 2002).

\subsection{Corpus de estudio}

El corpus de estudio está constituido por $60 \mathrm{CI}$, obtenidos de un total de 622 tesis y trabajos de grado, presentados en universidades hispanoamericanas durante el periodo 2000-2016. La tabla 1 muestra la distribución por países.

\begin{tabular}{ll}
\hline País & Frecuencia \\
\hline Venezuela & 81 \\
Perú & 96 \\
Chile & 51 \\
Ecuador & 78 \\
España & 60 \\
México & 100 \\
Guatemala & 96 \\
Argentina & 60 \\
Total & 622 \\
\hline
\end{tabular}

Tabla 1. Distribución del número de tesis según el país de origen.

Para seleccionar dicho corpus usamos los motores de búsqueda Google Académico y repositorios institucionales y universitarios hispanoamericanos con acceso gratuito a las versiones completas de las tesis. Empleamos estos descriptores: tesis, trabajo de grado, tesina, memoria de grado, odontología, estomatología, dental y dentista, combinados mediante el uso de los operadores lógicos $A N D$ y $O R$.

Desde el punto de vista cualitativo, se realizó un análisis textual manual del corpus. Tres investigadores independientes identificaron elementos, secciones, movimientos, pasos y realizaciones lingüísticas presentes en los CI, con el fin de identificar patrones.

Una vez identificados los patrones existentes en los documentos, entrevistamos a informantes expertos ${ }^{3}$ con el objeto de contrastar el análisis textual con el contextual, con las perspectivas de los informantes. El cuestionario empleado en dichas entrevistas fue validado por los tres especialistas y pilotado previamente. Con base a los testimonios de los informantes, intentamos explicar la presencia o ausencia de los rasgos discursivos más predominantes del corpus.

Desde el punto de vista cuantitativo, se realizó un análisis textual para identificar la frecuencia de aparición de los elementos y de los patrones de organización.

3 Un abogado, especialista en Odontología Social y legal, doctor en ciencias políticas y 9 Odontólogos investigadores, profesores, clínicos, autores de artículos, tutores, jurados evaluadores, todos con formación de maestría y doctorado. 


\section{Análisis de los resultados}

\subsection{Descripción del corpus}

Como ya se indicó, el corpus consta de 60 textos de CI incluidos en trabajos de grado y tesis, lo cual indica que solo cerca del $10 \%$ de las tesis anexa este tipo de documento. Coincidiendo con los informantes, el bajo número de documentos hallados puede deberse a dos factores: por un lado, las investigaciones experimentales con humanos, en las cuales es obligatorio emplear el CI, son escasas; predominan, en cambio, los estudios epidemiológicos. Por otro lado, debido a la falta de supervisión y vigilancia de las versiones que se publican en la web, algunos estudios experimentales no lo incluyen, lo cual coincide con Ariasgago y Marasso (2014). En la Tabla 2, se describen algunas características.

\begin{tabular}{|c|c|c|c|c|}
\hline Variable Documento & Doctorado & Maestría & Pregrado & Total \\
\hline $\mathrm{N}^{\circ}$ de textos & 3 & 6 & 51 & 60 \\
\hline$N^{0}$ de palabras & 763 & 1659 & 12166 & 14588 \\
\hline Media de palabras & 254,3 & 276,5 & 238,6 & 534,8 \\
\hline $\mathrm{N}^{\circ}$ de páginas & 4 & 7 & 52 & 63 \\
\hline Media páginas & 1,3 & 1,2 & 1,1 & 1,2 \\
\hline
\end{tabular}

Tabla 2. Descripción general del corpus.

Del total de los documentos analizados, 39 pertenecen a estudios realizados en Venezuela y los restantes, a estudios realizados en Argentina, Chile, Ecuador, España, Guatemala, México y Perú, como puede observarse en la Tabla 3.

\begin{tabular}{|c|c|c|c|c|}
\hline País Documento & Doctorado & Maestría & Pregrado & Total \\
\hline Venezuela & & 1 & 39 & 40 \\
\hline Perú & & 1 & 5 & 6 \\
\hline Chile & & 1 & & 1 \\
\hline Ecuador & & 2 & & 2 \\
\hline España & 3 & & & 3 \\
\hline México & & 1 & & 1 \\
\hline Guatemala & & & 4 & 4 \\
\hline Argentina & & & 3 & $\begin{array}{l}3 \\
60\end{array}$ \\
\hline
\end{tabular}

Tabla 3. Descripción del corpus según país de origen.

\subsection{Secciones que integran el consentimiento informado}

El análisis textual identifica cuatro secciones, como se muestra en la Tabla 4. A continuación se presenta la frecuencia de ocurrencia de cada sección.

\begin{tabular}{lll}
\hline Sección & \% & Frecuencia \\
\hline Identificación institucional & $60 \%$ & 36 \\
Título del documento & $100 \%$ & 60 \\
Cuerpo del documento & $100 \%$ & 60 \\
Cierre del documento & $100 \%$ & 60 \\
\hline
\end{tabular}

Tabla 4. Secciones que conforma el documento consentimiento informado. 


\subsubsection{Identificación institucional}

Esta sección registró una frecuencia de $60 \%$. Esto supone que los investigadores otorgan importancia a la información institucional asociada con la investigación. Según indicaron los informantes, es necesario proyectar la unidad académica en la que se realiza la investigación. La información institucional respalda la investigación y, a su vez, da a entender al participante que el proyecto ha sido previamente evaluado por los entes reguladores en la materia. Además, el hecho de que el participante tenga acceso a la información institucional podría generar mayor confianza y seguridad y, en consecuencia, aumentaría la posibilidad de que decida participar en la investigación.

En esta sección registramos la inclusión de forma recurrente de 4 elementos que se mencionan en la Tabla 5.

\begin{tabular}{lll}
\hline Elemento & \% & Frecuencia \\
\hline Logo de la institución & $40 \%$ & 24 \\
Nombre de la Universidad/Institución & $48 \%$ & 29 \\
Nombre de la facultad & $48 \%$ & 29 \\
Nombre del departamento & $35 \%$ & 21 \\
\hline
\end{tabular}

Tabla 5. Frecuencia de ocurrencia de los elementos de la sección A.

\section{Organización interna}

Hallamos que el 40\% de la información revisada de esta sección se organiza siguiendo esta ordenación:

1. Aparece el logo de la universidad, regularmente ubicado en la parte superior izquierda.

2. Al nivel del logo se ubica el nombre de la universidad a la cual está adscrito el programa educativo.

3. Debajo de la universidad, el nombre de la facultad/escuela a la cual está adscrito el programa educativo.

4. Se incluye el nombre del departamento donde se realiza la investigación.

5. Se incluye el nombre del proyecto.

Con base en la frecuencia de ocurrencia, se consideran obligatorias la inclusión del logo de la universidad/institución, el nombre de la universidad y el de la facultad/escuela adscrita al programa educativo, y serían opcionales la identificación del departamento en que se lleva a cabo la investigación, así como los datos de contacto de dicho departamento (número de teléfono, mail, dirección, entre otras).

\subsubsection{Título}

No encontramos uniformidad en el nombre que se le da al documento, como se muestra en la Tabla 6 . Sin embargo, "consentimiento informado" reportó la frecuencia más alta (65\%). Los informantes indicaron que esta es la denominación más apropiada, ya que identifica la función del documento. Skelton (1994) sugiere que un 
elemento puede ser considerado obligatorio 4 cuando reporta una frecuencia por encima del $65 \%$. Este resultado es coherente con los datos reportados en la literatura (Alfaro y García, 2011; Guirland, 2011; Oliva et al., 2011; Rodríguez, 2012; Ramírez et al., 2013; Escobar y Novoa, 2016):

\begin{tabular}{lll}
\hline Denominaciones & $\%$ & Frecuencia \\
\hline Carta de consentimiento & $11,67 \%$ & 7 \\
Consentimiento informado & $65 \%$ & 39 \\
Consentimiento de participación en el estudio & $6,67 \%$ & 4 \\
Consentimiento & $3,33 \%$ & 2 \\
Carta de consentimiento para el tratamiento & $5 \%$ & 3 \\
Consentimiento previo a la información & $1,67 \%$ & 1 \\
Ficha de consentimiento & $1,67 \%$ & 1 \\
Autorización & $5 \%$ & 3 \\
\hline
\end{tabular}

Tabla 6. Enunciados de la sección B presentados en porcentajes y ocurrencias.

\subsubsection{Cuerpo del documento}

Los informantes expertos coinciden en señalar que el documento CI debe estar escrito en un lenguaje llano, claro y conciso, que comunique, de forma clara, las condiciones del contrato, de modo de que pueda ser entendido por cualquier persona, en especial quienes no son conocedores de la disciplina odontológica. Esto implica evitar, en la medida de las posibilidades, usar términos especializados y ambiguos, y usar oraciones breves y ligeras.

\subsubsection{Movimientos retóricos}

El análisis del corpus arrojó como resultado la inclusión, en mayor o menor medida, de 5 movimientos retóricos en el cuerpo del documento, cuyas frecuencias se presentan codificadas en la Tabla 7.

\begin{tabular}{lll}
\hline Código & Nombre del movimiento & $\%$ \\
\hline M1 & Identificación de los involucrados en la investigación & 80 \\
M2 & $\begin{array}{l}\text { Descripción de la investigación } \\
\text { M3 }\end{array}$ & $\begin{array}{l}\text { Manifestación de haber sido informado sobre la naturaleza de la } \\
\text { investigación. }\end{array}$ \\
M4 & $\begin{array}{l}\text { Manifestación de haber comprendido la información sobre el } \\
\text { procedimiento clínico. }\end{array}$ & 72 \\
M5 & Ratificación del consentimiento. & 73 \\
\hline
\end{tabular}

Tabla 7. Movimientos discursivos pertenecientes a la sección C

El primer movimiento (Identificación de los involucrados en la investigación) se considera obligatorio con base a su frecuencia y a las opiniones de los informantes. Estos manifestaron que la inclusión de esta información debe ser ineludible debido al carácter contractual del CI; su ausencia quitaría legalidad al documento.

Para los informantes, la aparición del segundo movimiento (Descripción de la investigación) aporta confiabilidad, debido a que brinda al participante una idea clara sobre el estudio en el que se busca que participe. encima de $65 \%$. En cambio, se consideran opcionales aquellas secuencias cuya frecuencia de aparición sea superior al 50\%. Sin embargo, en los casos en los que no llega a este porcentaje, pero los informantes así lo consideren también se recomendará su inclusión. 
Esto coincide con los resultados del análisis textual, en el cual se encontró que el 100\% de los documentos incluyen esta información.

Coherente con la frecuencia, los informantes sugieren la inclusión obligatoria del tercer movimiento (Manifestación de haber sido informado sobre la naturaleza de la investigación). Ellos consideran que es totalmente necesario brindarle la información clara y pertinente sobre los beneficios, posibles riesgos y costos que puedan generar su participación en la investigación. Por ejemplo, un informante refirió que es esencial que el investigador aporte toda la información económica que involucre al participante.

El cuarto movimiento (Manifestación de haber comprendido la información sobre el procedimiento clínico), también debería ser obligatorio a causa de su frecuencia (72\%) y de las opiniones de los expertos. Con base al ordenamiento jurídico vigente en Venezuela, el CI debe incluir un apartado en el que el participante indique que ha comprendido la información pertinente al procedimiento clínico en el cual está basada la investigación. Los informantes indicaron que no es suficiente con informar; es necesario que el participante indique que ha comprendido dicha información.

Por último, el quinto movimiento (Ratificación del consentimiento) consiste, en la opinión de los expertos consultados, en una declaración manifiesta de aceptación, de forma explícita y voluntaria, de formar parte del estudio habiendo sido informado y habiendo comprendido la serie de procedimientos clínicos a los que se va a someter. Por esto y por la frecuencia de uso (73\%), este movimiento también debería ser obligatorio.

\subsubsection{Pasos en que se pueden realizar los movimientos}

Según los resultados, cada uno de estos movimientos se puede realizar mediante diferentes pasos 5 . Para efectos de esta investigación, siguiendo a Skelton (1994), los pasos son expresiones mediante las cuales se puede transmitir el contenido semántico del movimiento y cumplir su función retórica. Como se observa en la tabla 8 , se registraron un total de 16 pasos distribuidos de la siguiente manera: 4 en el primer movimiento; 8 en el segundo movimiento; 3 en el tercer movimiento y; 1 en el cuarto.

\subsubsection{Realizaciones de los pasos}

A continuación, se presentan las expresiones con que se materializan lingüísticamente los pasos de cada movimiento discursivo. Luego, con base en su frecuencia de uso, se determinan los marcadores discursivos correspondientes.

\begin{tabular}{lll}
\hline Movimientos y pasos & Frec. & \% \\
\hline M1 Identificación de los involucrados en la investigación & & \\
P1 Identificación de los participantes & 46 & $76,6 \%$ \\
P2 Identificación del representante legal & 8 & $13,3 \%$ \\
P3 Identificación del investigador (tesista, tutor) & 25 & $40 \%$ \\
P4 Identificación de asesores & 5 & $8,3 \%$ \\
M2 Descripción de la investigación & 51 & $85 \%$ \\
P1 Nombre del proyecto de investigación & & \\
\hline
\end{tabular}

5 En algunos documentos, un movimiento se puede realizar concretamente mediante la inclusión de un solo un paso; en otros, pueden haberse incluido simultáneamente todos los pasos posibles en dicho movimiento. 
P2 Objetivos de la investigación

P3 Descripción de aspectos metodológicos

P4 Alcance de la investigación

M3 Manifestación/declaración de haber sido informado

P1 Manifestación/declaración de haber sido informado sobre la naturaleza del estudio.

P2 Manifestación/declaración de haber sido informado sobre el procedimiento clínico (efectos adversos, posibles riesgos y complicaciones, riesgos asociados al uso de fármacos, riesgos asociados a maniobras quirúrgicas, beneficios del procedimiento clínico y pronósticos del tratamiento).

P3 Manifestación/declaración de haber recibido garantía de confidencialidad y anonimato.

P4 Manifestación/declaración de haber sido informado sobre el carácter voluntario de su participación en el estudio.

P5 Manifestación/declaración de haber sido informado de su derecho al retiro voluntario del estudio, en 27 cuyo caso se exime de responsabilidades a los investigadores.

P6 Manifestación/declaración de haber sido informado sobre las implicaciones económicas de la 28 participación en la investigación

P7 Manifestación/Declaración del compromiso de suministrar información veraz durante toda la 11 investigación.

P8 Manifestación/declaración de haber recibido garantía de que será informado veraz y oportunamente sobre 22 el desarrollo de los estudios que se le realizarán.
$11 \quad 18,3$

$54 \quad 90 \%$

$5 \quad 8,3 \%$

$25 \quad 41,6 \%$

$34 \quad 56,6 \%$

$51,6 \%$

$830 \%$

$45 \%$

$46,6 \%$

$18,3 \%$

$36,6 \%$

\section{M4 Manifestación/declaración de haber comprendido la información}

P1 Manifestación/declaración de haber comprendido la información sobre la investigación.

P2 Manifestación/declaración de haber comprendido la información sobre el procedimiento clínico

P3 Autorización para la publicación en medios científicos de los hallazgos clínicos asociados a los estudios 8 que le hayan realizado.

P4 Autorización para que se le realicen exámenes y tratamientos complementarios (estudios diagnósticos, 14 estudios clínicos, estudios paraclínicos, tratamientos alternativos asociados a potenciales complicaciones) si fueren necesarios.

\section{M5 Ratificación del consentimiento}

P1A Satisfacción con la información recibida.

P1B Aceptación de las condiciones de mi participación en la investigación.

P1C Aceptación de la participación en la investigación.

P2 Otorgamiento del consentimiento para que se realicen los estudios.
$15 \%$

$15 \%$

$13,3 \%$

$23,3 \%$

Tabla 8. Frecuencia de ocurrencia de los pasos de cada movimiento retórico de la sección C del documento consentimiento informado.

\section{Realizaciones de los pasos del movimiento 1}

A continuación, en el Cuadro 1, se presentan algunas expresiones mediante las cuales se realizan los pasos del movimiento 1 .

\begin{tabular}{|l|}
\hline M1 Identificación de los involucrados en la investigación \\
\hline P1 Identificación de los participantes \\
\hline R1 Yo, nombre y apellido del paciente \\
R2 Yo, nombre y apellido del paciente, C.I. del participante \\
R3 Yo, nombres y apellido del participante, datos demográficos, civiles y legales del participante \\
R4 Nombre y apellido del paciente \\
\hline P2 Identificación del representante legal \\
\hline R1 Yo, nombre y apellido del representante legal \\
\hline P3 Identificación del investigador (tesista, tutor) \\
\hline R1 Nombre y apellido del participante \\
\hline P4 Identificación de asesores \\
\hline R1 Nombre y apellido del(los) asesores \\
\hline
\end{tabular}

Cuadro 1. Realizaciones de los pasos del movimiento 1 . 
El análisis de las realizaciones de los pasos que integran el movimiento 1 coincide, en parte, con los resultados obtenidos en la literatura consultada (Alfaro y García, 2011; Oliva et al. 2011).

\section{Realizaciones de los pasos del movimiento 2}

En este movimiento, se suele citar literalmente los datos del proyecto: título, objetivos, metodología y alcances. Operativamente, según los informantes, la información escrita se debe complementar con explicaciones orales, con el apoyo audiovisual si fuere necesario. A continuación, en el Cuadro 2, se presentan algunas expresiones mediante las cuales se realiza el segundo movimiento.

\begin{tabular}{|l|}
\hline Descripción de la investigación \\
\hline P1 Nombre del proyecto de investigación \\
\hline R1 Usted ha sido convocado a participar en un estudio llamado \\
R2 Fui informado de mi participación en el estudio titulado \\
R3 He recibido información del trabajo titulado \\
R4 Entiendo que mi participación en el estudio consiste en \\
\hline P2 Objetivos de la investigación \\
\hline R1 He sido informado que este estudio tiene como objetivos \\
R2 La investigación se realiza con el propósito de \\
R3 El propósito del presente consentimiento es informarle al paciente que \\
R4 He sido informado que esta investigación pretende proponer \\
R5 La presente investigación consiste en \\
\hline P3 Descripción de aspectos metodológicos \\
\hline R1 En dicha investigación seré sometido a \\
R2 Entiendo que seré encuestado(a) en mi facultad \\
R3 Se le informa que su representado será sometido a una evaluación clínica \\
R4 Para ello se hará un examen de la cavidad bucal \\
\hline P4 Alcance de la investigación \\
\hline R1 La investigación proporcionará información valiosa para la educación y formación profesional de los futuros \\
dentistas
\end{tabular}

Cuadro 2. Realizaciones de los pasos del movimiento 2.

\section{Realizaciones de los pasos del movimiento 3}

Se registró un uso frecuente de un lenguaje especializado, por un lado, términos médicos y odontológicos para referirse a lesiones, tratamientos, procedimientos clínicos y metodológicos, riesgos y complicaciones; por el otro, expresiones jurídicas y legales para indicar derechos, garantías y compromisos que tiene el participante al acceder participar en el estudio. Contrario a los hallazgos del análisis textual, para los informantes es indispensable comunicar esta información evitando expresiones técnicas, obscuras, que obstaculicen la comprensión total. El Cuadro 3 presenta algunas expresiones mediante las cuales se realizan los pasos del tercer movimiento.

\begin{tabular}{|l|}
\hline M3. Manifestación/declaración de haber sido informado sobre la naturaleza de la investigación \\
\hline P1 Manifestación de haber sido informado sobre la naturaleza del estudio \\
\hline R1 Entiendo que mi participación en el estudio consiste en \\
R2 He sido informado de manera verbal y escrita que \\
R3 Se me ha explicado que \\
R2 El propósito del presente consentimiento es informarle al paciente que \\
\hline P2 Manifestación de haber sido informado sobre el procedimiento clínico (riesgos) \\
\hline Revista de Lenguas para Fines Específicos 26.1 \\
ISSN: $2340-8561$
\end{tabular}


R1 Entiendo que los procedimientos descritos no involucran ningún riesgo ni malestar para mi salud

R2 Conozco los posibles riesgos y complicaciones

R3 He sido informado que participar en dicha investigación no supone ningún riesgo para mi salud

R4 Pueden ocurrir ciertas molestias y efectos

P3 Manifestación de haber recibido garantía de confidencialidad y anonimato.

R1 Entiendo que los datos obtenidos por los investigadores son de carácter confidencial

$\mathbf{R} 2$ Los datos que yo proporcione serán confidenciales

R3 La investigación mantendrá mi anonimato

R4 Se me ha garantizado confidencialidad

R5 Le aseguramos mantener en estricta reserva su identidad

P4 Manifestación de haber sido informado sobre el carácter voluntario de su participación en el estudio.

R1 Su participación es completamente voluntaria

R2 Entiendo que mi participación en este estudio es voluntaria

R3 Usted puede decidir si participa o no

P5 Manifestación de haber sido informado de su derecho al retiro voluntario del estudio

R1 Puede retirarse del proyecto en cualquier momento

R2 Tiene derecho a retirarse sin que ello le afecte

$\mathbf{R 3}$ Usted puede retirarse del proyecto en cualquier momento

R4 Puedo dejar de participar en esta investigación en el momento que lo desee

P6 Manifestación de haber sido informado sobre las implicaciones económicas

R1 Confío en que los datos suministrados por mi serán utilizados sin fines de lucro ni intereses personales

R2 No he recibido ni pretendo algún beneficio económico por mi participación en el estudio

R3 Mi participación en el estudio no significará un gasto económico de ninguna índole

R4 No recibirá remuneraciones económicas ni su participación le generará costo alguno.

P7 Manifestación del compromiso de suministrar información veraz

R1 Los datos relativos a mi historia clínica son ciertos

$\mathbf{R 2}$ Me comprometo a proporcionar datos veraces y completos

R3 Doy fe de no haber omitido datos al exponer mi historial

P8 Manifestación de haber recibido garantía de que será informado veraz y oportunamente

R1 Seré informado en cada momento y a mi requerimiento la evolución del proceso

R2 Usted será informado de cualquier cambio que ocurriese durante el estudio

R3 Cualquier pregunta que tenga sobre el estudio me será respondida oportunamente

\section{Cuadro 3. Realizaciones de los pasos del movimiento 3}

El análisis de los pasos/realizaciones lingüísticas que integran el movimiento 3 coincide con los resultados obtenidos en estudios previos (Faden y Beauchamp, 1986; Revilla y Fuentes, 2007; Visbal, 2009; Abou, 2010).

\section{Realizaciones de los pasos del movimiento 4}

Similar a lo que se reportó en el movimiento 3, el uso de lenguaje especializado en este movimiento, presentado en $1^{a}$ persona, deja la impresión de que los participantes dominan este registro. De acuerdo con los informantes, si el CI, escrito con un lenguaje especializado, no se complementa con una explicación oral divulgativa, es probable que el participante certifique un contrato que no ha comprendido totalmente, lo cual es contrario al espíritu, razón y motivo del CI (Garbin et al., 2007; Abou, 2010; Roges et al., 2010). A continuación, se presentan algunas expresiones mediante las cuales se realizan los pasos del cuarto movimiento (Cuadro 4).

\begin{tabular}{|l|}
\hline M4. Manifestación/declaración de haber comprendido la información sobre el procedimiento clínico \\
\hline P1 Manifestación/declaración de haber comprendido la información sobre la investigación \\
\hline R1 Leí la información que se me entregó sobre el estudio \\
R2 Me han explicado el procedimiento \\
R3 Declaro tener conocimiento claro que el objetivo fundamental del trabajo es \\
\hline
\end{tabular}


R4 Al firmar este documento indico que he sido informado de la investigación

R5 He leído y se me ha informado sobre el propósito de esta investigación

P2 Manifestación/declaración de haber comprendido la información sobre el procedimiento clínico

R1 Comprendo que la intervención quirúrgica va a consistir en

R2 Declaro haber leído, comprendido y recibido las respuestas a mis preguntas respecto al estudio

R3 He sido informado(a) y comprendo la necesidad y fines de ser atendido

R4 Estoy enterado(a) de todo el examen y procedimiento que se me hará

P3 Autorización para la publicación en medios científicos de los hallazgos clínicos asociados a los estudios que le hayan realizado

R1 Doy consentimiento para la publicación de los datos obtenidos

R2 Doy consentimiento para la publicación de las fotografías clínicas con carácter científico

R3 Estoy de acuerdo con el uso para fines académicos de los resultados obtenidos

P4 Autorización para que se le realicen exámenes y tratamientos complementarios

R1 Doy mi consentimiento para ser fotografiado(a) durante y después de la intervención

R2 Si surgiese cualquier situación desesperada durante el tratamiento autorizo que se realice cualquier procedimiento o maniobra distinta de las usuales

R3 Usted deberá acudir a controles clínicos y radiográficos para valorar su evolución con el tratamiento

R4 Me comprometo a cumplir con las indicaciones pre y postoperatorias dadas por los responsables del

procedimiento

Cuadro 4. Realizaciones de los pasos del movimiento 4

Algunos de los estudios citados coinciden en identificar al paso 2 del movimiento 4 como un elemento fundamental previo a la ratificación del consentimiento informado (Oneyeaso y Utomi, 2007; Abou, 2010; Oliva et al., 2011; Oppliger y Bascuñan, 2011; Nouri y Rudd, 2015; Firdouse et al., 2017).

\section{Realizaciones de los pasos del movimiento 5}

Se registró el predominio de la $1^{\circ}$ persona del singular en todos los pasos. Esto implica que el CI, en vez de contrato entre dos partes (Garbin et al., 2007; Abou, 2010; Roges et al., 2010), es una declaración unilateral de parte del participante. A continuación, se presentan algunas expresiones mediante las cuales se realiza el movimiento 5. Es preciso señalar que contempla un solo paso que puede realizarse mediante 4 variantes, mutuamente excluyentes.

\begin{tabular}{|l|}
\hline M5. Ratificación del consentimiento \\
\hline P1A Satisfacción con la información recibida \\
\hline R1 Estoy satisfecho(a) con la información recibida \\
\hline P1B Aceptación de las condiciones de mi participación en la investigación. \\
\hline R1 Su firma indica que ha leído lo que se informa en esta hoja y sé que ha decidido participar en el estudio \\
R2 Acuerdo aceptar las condiciones de mi participación y a la vez autorizar al equipo de investigadores a realizar \\
el referido estudio \\
R3 Declaro aceptar voluntariamente ser parte de esta investigación \\
\hline P1C Aceptación de la participación en la investigación. \\
\hline R1 Acepto formar parte de un trabajo especial de grado \\
R2 Acepto libre y voluntariamente participar en este estudio \\
R3 Yo estoy de acuerdo en participar como individuo en este proyecto \\
R4 Su firma indica que ha leído lo que se informa en esta hoja y sé que ha decidido participar en el estudio \\
\hline P2 Otorgamiento del consentimiento para que se realicen los estudios. \\
\hline R1 Otorgo mi consentimiento informado para que sea realizado el procedimiento a mi representado \\
R2 Consiento participar en el estudio \\
R3 Por la presente autorizo la participación en el estudio \\
\hline
\end{tabular}

Cuadro 5. Realizaciones de los pasos del movimiento 5. 
González, Rodríguez y Berro (2005) identificaron pasos similares del movimiento $\mathrm{N}^{\circ} 5$ en el cuerpo del documento.

\subsubsection{Cierre del documento}

Se identificaron algunos elementos usados para cerrar el documento, según los informantes expertos, para darle legalidad al contrato que supone el CI. Entre éstos cabe destacar que, por la frecuencia de ocurrencia, podrán ser considerados obligatorios la firma del participante y la indicación del lugar y la fecha de firma. Sin embargo, en su condición de acuerdo contractual, legalmente se requiere incluir la firma del investigador, aunque su frecuencia fue de $50 \%$.

La tabla 9 presenta la lista de dichos elementos con sus respectivas frecuencias de ocurrencia, para recomendar cuáles serían obligatorios y cuáles, opcionales.

\begin{tabular}{lll}
\hline Nombre del elemento & \% & Frecuencia \\
Firma del participante (manuscrito) & 80 & 48 \\
Firma del representante legal del participante (manuscrito) & 17 & 10 \\
Firma del investigador (manuscrito) & 50 & 30 \\
Firma del testigo (manuscrito) & 10 & 6 \\
Firma de asesores de la investigación (manuscrito) & 18 & 11 \\
$\mathrm{~N}^{\circ}$ de documento de identidad del paciente (manuscrito) & 30 & 18 \\
$\mathrm{~N}^{\circ}$ de documento de identidad del investigador (manuscrito) & 10 & 6 \\
Lugar y fecha (manuscrito) & 87 & 52 \\
Contactos del paciente o del representante legal & 10 & 6 \\
Información institucional en formato de pie página & 7 & 4 \\
\hline
\end{tabular}

Tabla 9. Frecuencia de los elementos de la sección D.

Kvitko y Carrillo (2012) encontraron que la sección de cierre del documento sería obligatoria en el documento de CI. Esto es coherente con los datos que arrojó nuestro análisis textual y con lo expresado por los expertos, quienes manifestaron que entre los elementos de cierre del documento es imprescindible incluir las firmas de las partes que integran la investigación (participante, investigador) y el lugar y fecha en los que se convino el CI, con la finalidad de darle un valor jurídico.

\section{Propuesta}

De acuerdo con el carácter jurídico que conlleva la participación de seres humanos en investigaciones experimentales, consideramos obligatorio incluir el documento de consentimiento informado como una práctica rutinaria propia de este tipo de estudios.

En las investigaciones odontológicas que cuenten con la participación de seres humanos no debería emplearse un formato de CI genérico. Por lo contrario, debería particularizarse a las características ajustadas del estudio y, a su vez, a los rasgos propios de los participantes.

Con fines de validar legalmente el documento de consentimiento informado, consideramos fundamental que se mantenga el carácter contractual de dicha práctica (Garbin et al., 2007; Abou, 2010; Roges et al., 2010); por lo tanto, ambas partes, el paciente/participante de la investigación (o su representante legal, cuando lo amerite el caso) y el clínico/investigador deben incluirse y conservar una versión original del documento a manera de respaldo legal. En este sentido, se recomienda hacer dos originales del mismo tenor. 
El documento debe ser escrito de forma clara, empleando un lenguaje llano, claro y conciso, que puedan ser entendidos por cualquier persona, en especial quienes no son conocedores de la disciplina odontológica. Esto implica evitar usar términos especializados y ambiguos. Usar oraciones breves y ligeras.

Con base al análisis textual, siguiendo las recomendaciones de los informantes expertos, y en vista de que no se dispone de modelos válidos de consentimientos informados para ser empleados en la investigación odontológica, en el siguiente cuadro presentamos un modelo de consentimiento informado. Consideramos que este formato puede ser utilizado como guía para la enseñanza de la investigación, pues es coherente con lo aceptado por la comunidad internacional dentro del marco jurídico vigente.

\begin{tabular}{|c|c|}
\hline \multicolumn{2}{|c|}{ Identificación institucional } \\
\hline \multirow{4}{*}{ 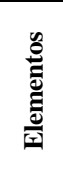 } & Logo de la universidad/institución \\
\hline & Nombre de la universidad/institución \\
\hline & Nombre de la Facultad \\
\hline & Nombre del Departamento \\
\hline \multicolumn{2}{|r|}{ (Título del documento) Consentimiento informado } \\
\hline \multicolumn{2}{|c|}{ Cuerpo del documento } \\
\hline \multirow{5}{*}{ 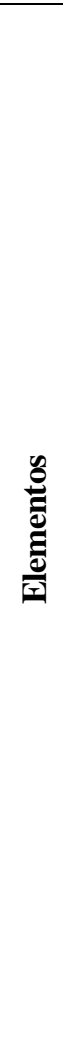 } & $\begin{array}{l}\text { Identificación de los involucrados en la investigación } \\
\text { Identificación del participante (y del representante legal cuando aplique) } \\
\text { Identificación del investigador }\end{array}$ \\
\hline & $\begin{array}{l}\text { Descripción de la investigación } \\
\text { Nombre del proyecto de investigación } \\
\text { Descripción de aspectos metodológicos }\end{array}$ \\
\hline & $\begin{array}{l}\text { Manifestación de haber sido informado sobre la naturaleza de la investigación } \\
\text { Manifestación de haber sido informado sobre la naturaleza del estudio. } \\
\text { Manifestación de haber sido informado sobre el procedimiento clínico (efectos adversos, posibles riesgos } \\
\text { y complicaciones, riesgos asociados al uso de fármacos, riesgos asociados a maniobras quirúrgicas, } \\
\text { beneficios del procedimiento clínico y pronósticos del tratamiento). } \\
\text { Manifestación de haber recibido garantía de confidencialidad y anonimato. } \\
\text { Manifestación de haber sido informado sobre el carácter voluntario de su participación en el estudio. } \\
\text { Manifestación de haber sido informado de su derecho al retiro voluntario del estudio, en cuyo caso se } \\
\text { exime de responsabilidades a los investigadores. } \\
\text { Manifestación de haber sido informado sobre las implicaciones económicas de la participación en la } \\
\text { investigación. }\end{array}$ \\
\hline & $\begin{array}{l}\text { Manifestación de haber comprendido el procedimiento clínico } \\
\text { Manifestación de haber comprendido la información sobre la naturaleza de la investigación. } \\
\text { Manifestación de haber comprendido la información sobre el procedimiento clínico. } \\
\text { Autorización para la publicación en medios científicos de los hallazgos clínicos asociados a los estudios } \\
\text { que le hayan realizado. } \\
\text { Autorización para que se le realicen exámenes y tratamientos complementarios si fueren necesarios. }\end{array}$ \\
\hline & $\begin{array}{l}\text { Ratificación del consentimiento } \\
\text { Aceptación de la participación en la investigación. } \\
\text { Otorgamiento del consentimiento para que se realicen los estudios. }\end{array}$ \\
\hline \multicolumn{2}{|c|}{ Cierre del documento } \\
\hline \multirow{5}{*}{ 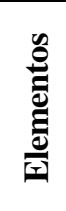 } & Nombre y apellido del participante \\
\hline & Firma del participante (y del representante legal cuando aplique) \\
\hline & Nombre y apellido del investigador \\
\hline & Firma del investigador, núm. de registro del Ministerio de Salud y del colegio profesional respectivo. \\
\hline & Lugar y fecha \\
\hline
\end{tabular}

Cuadro 6. Propuesta de formato de consentimiento informado. 


\section{Conclusiones}

La frecuencia de inclusión del CI en las tesis y trabajos de grado, como documento legal previo a la ejecución de las terapias/maniobras clínicas propias de los estudios experimentales con humanos, fue baja, lo cual contraría lo recomendado por los informantes. Esto sugiere que en la práctica se le resta importancia al papel que juega este documento en la investigación odontológica, o no se hace pública, lo cual amerita una investigación más profunda.

Con base en el análisis textual y el testimonio de los informantes, se encontraron coincidencias en las secciones que integran el CI y a sus respectivos elementos, y en las secuencias como se organizan las secciones del documento y los elementos de cada sección.

El CI es un documento personal, que debería incluir solo la información referida al tipo de procedimiento o tratamiento a realizar; por lo tanto, deben evitarse documentos que incluyan textos genéricos o que parezcan una reproducción del consentimiento empleado para la práctica eminentemente clínica. Esto evidencia la necesidad de reformular el texto del documento con los rasgos prototípicos ideales en relación con la importancia que posee este proceso.

Los documentos de CI utilizados con fines investigativos cumplen la función de informar y facilitar la comunicación entre el investigador y el paciente. Por ello, hacen falta documentos escritos en lenguaje claro, plano y comprensible para lograr que puedan generar satisfacción, claridad y confianza.

Por otro lado, similar a lo reportado por García et al. (2011) y Quintana (2009), en las tesis y TEG analizadas, hallamos que los estudios experimentales no suelen anexar el documento CI. Esto limitó el número de textos incluidos en el corpus y, en consecuencia, la posibilidad de generalizar a partir de los resultados. Esto permite pensar que se desconoce la importancia de hacer público este documento, el cual además es un requisito ético y legal. Sin embargo, sería interesante estudiar esta práctica en futuras investigaciones.

Finalmente, sugerimos emplear el formato propuesto como guía para la enseñanza de la redacción y uso del CI en las clases de escritura académica e investigación. Esto implica, sin embargo, complementar el formato aquí propuesto con las perspectivas de los miembros establecidos de la comunidad y con la evidencia derivada de posteriores análisis discursivos del consentimiento usado para fines investigativos.

\section{About the authors}

Jesús Reinaldo Arias: Odontólogo egresado de la Facultad de Odontología de la Universidad de Los Andes, Mérida, Venezuela. Práctica clínica privada en Mérida, Venezuela y Buenos Aires, Argentina. Desde el 2016, participa en las actividades clínicas, educativas y de investigación como parte del Programa Itinerante de Atención a Poblaciones Dispersas de la ULA, prestando atención odontológica a indígenas del Amazonas Venezolano y a otras poblaciones de bajos recursos.

André José Caldera: Odontólogo egresado de la Facultad de Odontología de la Universidad de Los Andes, Mérida, Venezuela. Práctica clínica privada Práctica clínica privada en Mérida, Venezuela y Buenos Aires, Argentina. Desde el 2014, participa en las actividades clínicas, educativas y de investigación como parte del 
Programa Itinerante de Atención a Poblaciones Dispersas de la ULA, prestando atención odontológica a indígenas del Amazonas Venezolano y a otras poblaciones de bajos recursos.

Oscar Alberto Morales: Máster en Lectura y Escritura, Máster en Lingüística y Doctor en "Comunicación Multilingüe (Universidad Pompeu Fabra, España). Profesor titular jubilado de Lectura y Escritura e Investigación, Facultad de Odontología, Universidad de Los Andes. Profesor de tiempo parcial, Carrera de Odontología, Universidad de los Hemisferios. Editor de La Revista Venezolana de Investigación Odontológica de la IADR. Autor de más de 100 artículos y conferencias sobre lectura, escritura y discurso académico.

Bexi Perdomo: Magíster en Educación, mención Enseñanza de Inglés como Lengua Extranjera. Candidata a doctora del Ciencias Humanas de la ULA. Miembro del Grupo Multidisciplinario de Investigaciones en Odontología (G-MIO). Profesora de Inglés con Propósitos Odontológicos e Investigación en la Facultad de Odontología de la Universidad de Los Andes, Venezuela

Daniel Cassany: Doctor en Filosofía y Letras, profesor titular de Análisis del Discurso en la Universitat Pompeu Fabra, coordinador del Grupo de investigación Literacidad Crítica, autor de más de una decenas de libros y más de 100 artículos y conferencias sobre lectura, escritura y discurso.

Élix Izarra: Licenciado en Estadística, Magíster en Estadística Aplicada al Sector Público, Universidad Alcalá de Henares, Madrid, España. Profesor de Bioestadística en la Facultad de Odontología de la Universidad de Los Andes, Venezuela. Consultor estadístico de Organizaciones Internacionales Multilaterales, e instituciones públicas y privadas.

\section{Acknowledgements}

Este artículo muestra resultados parciales de un proyecto de investigación financiado por el Consejo de Desarrollo Científico, Humanístico, Tecnológico y de las Artes de la Universidad de Los Andes, Mérida, Venezuela, código O-307-15-06-A.

Para su desarrollo, también recibimos el apoyo de la Fundación Carolina, España, a través de una beca de Postdoctorado en la Universitat Pompeu Fabra, Barcelona, España, para el periodo 2017-2018.

\section{Referencias}

Abou, R. (2010). El odontólogo frente al consentimiento informado. Aspectos relevantes. Acta Odontológica Venezolana, 49(2).

Alfaro,L. \& García,C. (2011). Percepción del proceso de consentimiento informado en pacientes de la Clínica Estomatológica Central de la Universidad Peruana Cayetano Heredia. Revista Estomatológica Herediana, 21(1), 5-12.

Ariasgago, O. \& Marasso, S. (2014). El consentimiento informado en las publicaciones de investigación odontológica. Acta Odontológica Venezolana, 52(3), 1-9.

Baú, M. (2000). Capacidad e Jurídica e consentimento informado. Bioética, 8(2), 285-296.

Bhatia, V. K. (2002). Applied genre analysis: A multi-perspective model. Ibérica, 4, 3-19. 
Brehaut, J. C., Carroll, K., Elwyn, G., Saginur, R., Kimmelman, J., Shojania, K., \& Fergusson, D. (2015). Elements of informed consent and decision quality were poorly correlated in informed consent documents. Journal of Clinical Epidemiology, 68(12), $1472-1480$. https://doi.org/10.1016/j.jclinepi.2015.03.002 [retrieved: 11.3.2018]

Brítez, S. (2001). Conocimientos, actitudes y prácticas sobre el Código de Ética Odontológica en odontólogos de la Policía Nacional en el año 2009. Memorias del Instituto de Investigaciones en Ciencias de la Salud, 7(1), 26-34.

Brito, N. \& Corral, Y. (2014). La historia clínica y el consentimiento informado en investigaciones clínicas y odontológicas. Acta Odontológica Venezolana, 52(2), 1-24.

Castro Maldonado, B., Callirgos Lozada, C. C., Rojas, F., Efraín, V., Leguía Cerna, J. A., \& Díaz-Vélez, C. (2010). Evaluación de la calidad de estructura y contenido de los formatos de consentimiento médico informado de los hospitales de la Región Lambayeque. Marzo-junio 2010. Acta Médica Peruana, 27(4), 238-243.

Correia, C. D. C., \& Silva, J. J. D. (1999). Pesquisa em seres humanos: Aspectos Bioéticos. An. Fac. Odontol. Univ. Fed. Pernamb., 9(1), 57-9.

Doyal, L. \& Cannell, H. (1995). Informed consent and the practice of good dentistry. Br Dent Journal, 178(12), 454-460. https://doi.org/10.1038/sj.bdj.4808802 [retrieved: 17.8.2018]

Duque, J. (2011). El Consentimiento informado ¿Cuestión de formalismo legal o cuestión de humanidad? Revista Médico-legal, 1(3), 31-33.

Escobar, M. \& Novoa, E. (2016). Análisis de formatos de consentimiento informado en Colombia. Problemas ético-legales y dificultades en el lenguaje. Revista Latinoamericana de Bioética, 16(1), 14-37.

Faden, R. R. \& Beauchamp, T. L. (1986). A history and theory of informed consent. Londres: Oxford University Press.

Falagas, M. E., Korbila, I. P., Giannopoulou, K. P., Kondilis, B. K., \& Peppas, G. (2009). Informed consent: how much and what do patients understand? The American Journal of Surgery, 198(3), 420-435. https://doi.org/10.1016/j.amjsurg.2009.02.010 [retrieved: 27.6.2018]

Ferro, M. (2014). Consentimiento informado en menores de edad del Centro de Atención Odontológica al Paciente con Discapacidad (CAPDIS). Facultad de Odontología. Universidad Central de Venezuela. Acta Odontológica Venezolana, 52(4).

Firdouse, M., Wajchendler, A., Koyle, M., \& Fecteau, A. (2017). Checklist to improve informed consent process in pediatric surgery: A pilot study. Journal of Pediatric Surgery, 52(5), 859-863. https://doi.org/10.1016/j.jpedsurg.2017.01.023 [retrieved: 21.5.2018]

Garbín C, Garbín A, Santos, C. y Goncalves, P (2009). Percepción del cirujano-dentista respecto del uso del consentimiento informado en el tratamiento odontológico. Acta bioethica, 15(1), 106-111.

Garbin, C. A. S., Garbin, A. J. I., Saliba, N. A., Santos, C. D., \& Gonçalves, P. E. (2009). Percepción del cirujano-dentista respecto del uso del consentimiento informado en el tratamiento odontológico. Acta Bioethica, 15(1), 106-111.

Garbin, C. A. S., Garbin, A. J. I., Saliba, N. A., Zina, L. G., \& Gonçalves, P. E. (2007). El consentimiento informado en la clínica odontológica. Acta Odontológica Venezolana, 45(1), 37-43.

García González, M. C., Tarajano Roselló, A. O., Rosabales Quiles, I., \& Ortega González, N. (2011). Comunicación y consentimiento informado en servicios de Ortopedia y Neurocirugía del Hospital Provincial de Camagüey. Humanidades Médicas, 11(1), 81-98.

González, D. Rodríguez, H y Berro, G. (2005). Consentimiento informado. Análisis crítico de su aplicación en un servicio quirúrgico. Revista Médica Uruguaya, 21, 291-297.

Grez, L. (1999). Ética en la atención de urgencia. Rev. Chile. Pediatría, 70(4), 12-15.

Guirland, A. (2011). Percepción y comprensión del consentimiento informado en pacientes que acudieron a la facultad de odontología en el año 2010-2011. Memorias del Instituto de Investigaciones en Ciencias de la Salud, 9(2), 43-49.

Gupta, V. V., Bhat, N., Asawa, K., Tak, M., Bapat, S., \& Chaturvedi, P. (2015). Knowledge and attitude toward informed consent among private dental practitioners in Bathinda city, Punjab, India. Osong public health and research perspectives, 6(2), 73-78. https://doi.org/10.1016/j.phrp.2014.12.005 [retrieved: 11.5.2018]

Kvitko, L y Carrillo, R. (2012). Aspectos interesantes sobre el consentimiento informado en la práctica médica. Boletín Médico, 2(12).

Málaga Rodríguez, G., Tupayachi Ortiz, G., Guevara Guevara, T., \& Hidalgo Salinas, F. (2007). Evaluación de la calidad de los consentimientos informados de los servicios de los hospitales de nivel III-IV de Lima y Callao. Revista Médica Herediana, 18(3), 136-142. 
Maluf, F., Carvalho, G. P. D., Diniz Júnior, J. C., Bugarin Junior, J. G., \& Garrafa, V. (2007). Consentimento livre e esclarecido em odontologia nos hospitais públicos do Distrito Federal. Ciência \& Saúde Coletiva, 12, 1737-1746.

Marchi, M. \& Sztajn, R. (1998). Autonomia e Heteronomia na relação entre profissionais de saúde e usuário dos serviços de saúde. Bioética, 61, 39-45.

Miguel, R., \& Zemel, M. (2006). El Consentimiento Informado en odontólogos residentes de la Facultad de Odontología de la Universidad Nacional de la Plata. Acta bioethica, 12(1), 81-89.

Nouri, S. \& Rudd, R. (2015). Health literacy in the oral exchange: An important element of patient-provider communication. Patient Education and Counseling, 98(1), 565-571. https://doi.org/10.1016/j.pec.2014.12.002 [retrieved: 11.12.2017]

Oliva, J. E., Bosch, C., Carballo, R. \& Fernández J. E. (2011). El consentimiento informado, una necesidad de la investigación clínica en seres humanos. Revista Cubana de Investigaciones Biomédicas, 20(2), 150-158.

Olufowote, J. O. (2008). A structurational analysis of informed consent to treatment: Societal evolution, contradiction, and reproductions in medical practice. Health communication, 23(3), 292-303. https://doi.org/10.1080/10410230802056404 [retrieved: 11.1.2018]

Oneyeaso, C. \& Utomi, I. (2007). Informed Consent in Orthodontics: Experiences of Nigerian Patients. Hellenic Orthodontic Review, 10(1), 29-39.

Oppliger, W. \& Bascuñan, L. (2011). Consentimiento informado. Percepción de médicos, enfermeras y padres sobre el proceso comunicativo. Revista Chilena de Pediatría, 82(3), 204-210.

Palomer, L. (2009). Consentimiento Informado en odontología. Un análisis teórico-práctico. Acta Bethic., 15 (1), $100-105$.

Pascucci, P. \& Travieso, G. (2008). La ética en el ejercicio de la Odontología. Mérida: Consejo de Publicaciones de la Universidad de Los Andes.

Pawlak, C. E., Fields, H. W., Beck, F. M., \& Firestone, A. R. (2015). Orthodontic informed consent considering information load and serial position effect. American Journal of Orthodontics and Dentofacial Orthopedics, 147(3), 363-372.

Quintana, J. (2009). El consentimiento informado en la publicación científica de Estomatología. Revista de Ciencias Médicas La Habana, 21(2), 332-334.

Quintero, H., Yáñez, M., Berríos, M., \& Morales, O. A. (2016). El consentimiento informado en la Práctica Odontológica privada del municipio Libertador de la ciudad de Mérida en Venezuela. Revista Odontológica de Los Andes, 11(2), 16-26.

Ramírez-Puerta, M. R., Fernández-Fernández, R., Frías-Pareja, J. C., Yuste-Ossorio, M. E., Narbona-Galdó, S., \& Peñas-Maldonado, L. (2013). Análisis de legibilidad de consentimientos informados en cuidados intensivos. Medicina Intensiva, 37(8), 503-509.

Revilla Lazarte, D. E., \& Fuentes Delgado, D. J. (2007). La realidad del consentimiento informado en la práctica médica peruana. Acta Médica Peruana, 24(3), 223-228.

Rodríguez, A. (2012). El uso del consentimiento informado por parte de los profesionales de salud en la atención clínico asistencial. Revista Electrónica Enfermería Actual en Costa Rica, 22, 1-11.

Rogés Sánchez, A. V., Sánchez García, S., Sanabria Negrín, J. G., Sosa Hernández, H., \& Moleiro Hernández, M. (2010). Aplicación del consentimiento informado por estomatólogos en la Atención Primaria de Salud. Revista de Ciencias Médicas de Pinar del Río, 14(1), 26-36.

Skelton, J. (1994). Analysis of structure of original research papers: an aid to writing original papers for publication. Br J Gen Pract., $44,455-459$.

Tahir, S., Ghafoor, F., Nusarat, S., \& Khan, A. (2009). Perception of consent among dental professionals. Journal of Medical Ethics and History of Medicine, 2(20). https://www.ncbi.nlm.nih.gov/pmc/articles/PMC3713957/[retrieved: 7.2.2018]

Taiwo, O. \& Kass, N. (2009). Post-consent assessment of dental subjects understanding of informed consent in oral health research in Nigeria. BMC Medical Ethics, 10(1), 1-7.

Visbal, G. (2009). Capacidad para tomar decisiones frente al consentimiento informado de personas que potencialmente participarían en estudios clínicos experimentales para la industria farmacéutica. Revista Científica Salud Uninorte, 26(1), 1-11.

Zurita, G. M., Hernández, J. A. S., Ruiz, M. T. R., Tapia, J. A. R., \& López, E. M. (2008). Comprensión del consentimiento informado en pacientes que requieren extracción del tercer molar. Revista CONAMED, 13(1), 24-28. 